\title{
ON CONCENTRATION DEPENDENCE OF SPIN-GLASS FREEZING TEMPERATURE IN Mn-ALLOYED II-V COMPOUNDS
}

\author{
H. Bednarski and J. Cisowski \\ Department of Solid State Physics, Polish Academy of Sciences \\ Wandy 3, 41-800 Zabrze, Poland
}

\begin{abstract}
Basing on recent studies of the magnetic properties of $\left(\mathrm{Zn}_{1-x} \mathrm{Mn}_{x}\right)_{3} \mathrm{As}_{2}$ (ZMA) and $\left(\mathrm{Cd}_{1-x} \mathrm{Mn}_{x}\right)_{3} \mathrm{As}_{2}$ (CMA) as well as on theoretical works devoted to the $\mathrm{Mn}-\mathrm{Mn}$ interaction in semimagnetic semiconductors, we have determined the distance dependence of this interaction taking into account the complicated crystal structure of both systems. The resulting radial dependence of the exchange interaction (being a sum of superexchange and the Bloembergen-Rowland mechanism) together with the scaling analysis have allowed us to describe the Mn-concentration dependence of spin-glass freezing temperature in ZMA, CMA and, for comparison, also in $\mathrm{Cd}_{1-x} \mathrm{Mn}_{x} \mathrm{Te}$ in very good agreement with experimental data.
\end{abstract}

PACS numbers: $75.50 . \mathrm{Lk}$

By alloying the $\mathrm{II}-\mathrm{V}$ tetragonal semiconducting compounds $\mathrm{Zn}_{3} \mathrm{As}_{2}$ and $\mathrm{Cd}_{3} \mathrm{As}_{2}$ with manganese, the $\left(\mathrm{Zn}_{1-x} \mathrm{Mn}_{x}\right)_{3} \mathrm{As}_{2}$ (ZMA) and $\left(\mathrm{Cd}_{1-x} \mathrm{Mn}_{x}\right)_{3} \mathrm{As}_{2}$ (CMA) systems, belonging to the family of semimagnetic semiconductors (SMSC's), can be formed. Extensive studies of the magnetic properties of both materials have been performed by Denissen et al. [1, 2], who analysed the experimental data within an extended version of the pair approximation. This analysis was based on an idealised quasi-cubic crystal structure of the systems in question, i.e. the $\mathrm{Mn}$ ions were assumed to be randomly located on an ideal simple cubic cation sublattice, what implies only one nearest-neighbour (NN) $\mathrm{Mn}-\mathrm{Mn}$ distance with a corresponding exchange constant $J_{1}$, a unique next-nearest-neighbour (NNN) distance with $J_{2}$, etc. In order to get a fair agreement between theory and experiment, a number of exchange constants as fitting parameters had to be introduced (for ZMA $J_{1}, J_{2}$ and $J_{3}$, see Fig. 1), completed with two additional parameters $J_{0}$ and $p$ describing the long-range interaction as $J_{\nu}=J_{0} R_{\nu}^{-p}$ for $\nu>3$; this type of interaction at larger distances has been deduced from a dependence of the spin-glass-paramagnet transition temperature $T_{\mathrm{f}}$ on $\mathrm{Mn}$ - concentration $[1,2]$.

Recently, de Vries et al. [3] carried out the detailed structural study of ZMA and found that the cation sublattice of this system is severely distorted, which results in 12 different values for the NN Mn-Mn distance, instead of one as assumed 


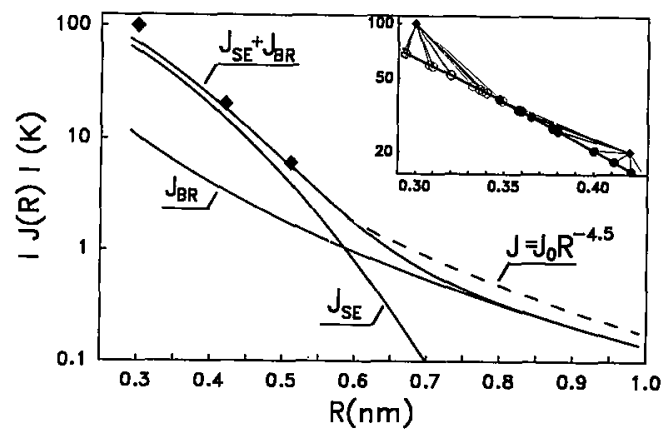

Fig. 1. Distance dependence of the exchange interaction in $\left(\mathrm{Zn}_{1-x} \mathrm{Mn}_{x}\right)_{3} \mathrm{As}_{2}$ with $x=0.08$. Solid lines represent theoretical dependence with the real crystal structure taken into account. Full rhombs and the dashed line are the result of a fitting procedure (Ref. [1]) for an idealised quasi-cubic structure. The inset shows the $J(R)$ dependence at short distances in greater detail and, additionally, a comparison between the cation-cation distances in the real structure (circles) and quasi-cubic structure (rhombs).

in $[1,2]$; similarly, the number of NNN distances increases considerably (see the inset in Fig. 1). Moreover, the first NN separation $R_{1}=0.29 \mathrm{~nm}$ in ZMA is, to our knowledge, the smallest one among the known SMSC's. Thus, Mn-alloyed II-V SMSC's appear to be good candidates to examine the radial dependence of the exchange constants, since we are dealing with a quasi-continuous spectrum of possible $\mathrm{Mn}-\mathrm{Mn}$ distances.

As follows from the theoretical studies of the magnetic interactions in SMSC's [4], there are two essential exchange mechanisms between the Mn-ions in these materials: (i) superexchange, which may be written as

$$
J_{\mathrm{SE}}(r)=J_{\mathrm{SE}}(r=1) \exp \left[-\alpha_{\mathrm{SE}}\left(r^{2}-1\right)\right]
$$

where $r=R / R_{1}$ is a distance between the Mn-ions (in units of the first NN distance $R_{1}$ ) and $\alpha_{\mathrm{SE}}$ is a constant [4] and (ii) the Bloembergen-Rowland (BR) exchange, which may be described as $J_{\mathrm{BR}}(r)=J_{\mathrm{BR}}(r=1) \exp \left[-\alpha_{\mathrm{BR}}(r-1)\right] / r^{3}$, where $\alpha_{\mathrm{BR}}$ is a constant $\propto \sqrt{E_{\mathrm{g}}}$ with $E_{\mathrm{g}}$ being the energy gap [5].

Basing on this approach and taking into account the real crystal structure of ZMA [3], we have obtained the $J(R)$-dependence $\left(J(R)=J_{\mathrm{SE}}(R)+J_{\mathrm{BR}}(R)\right)$, as shown in Fig. 1, by fitting the theoretical dependence to experimental results concerning the magnetic specific heat, magnetization and susceptibility [1, 2]. Having determined $J(R)$ [6], we have applied the scaling analysis allowing one to relate the spin-glass freezing temperature $T_{\mathrm{f}}$ to the Mn-concentration $x[7,8]$.

Our calculated curves along with the experimental data are shown in Fig. 2. Apart from ZMA and CMA, we present for comparison also the results obtained for $\mathrm{Cd}_{1-x} \mathrm{Mn}_{x} \mathrm{Te}$ (CMT) [9]. It can be seen that, in spite of an exponential character of the formulae describing both superexchange and the BR mechanism, the resulting curves, which very well reproduce the experimental data, are quite close to straight lines obtained when adopting a phenomenological power-law dependence of the type $J(R) \propto R^{-p}[7,8]$ which, however, has not any theoretical background. 


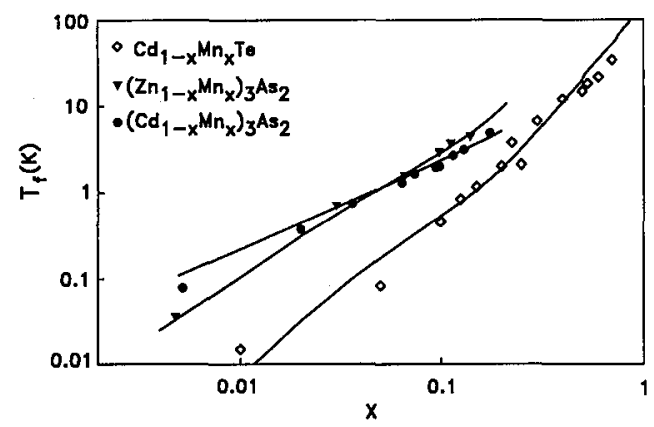

Fig. 2. Spin-freezing temperature as a function of the Mn concentration. The experimental data are taken from Refs. [1, 2] and [9] for ZMA, CMA and CMT, respectively. Solid lines are calculated with the following sets of parameters (all values of the first NN exchange constants $J^{1}=J(r=1)$ are negative and given in $\mathrm{K}$, while the exponential factors $\alpha$ are dimensionless): $J_{\mathrm{SE}}^{1}=63.5, \alpha_{\mathrm{SE}}=1.37, J_{\mathrm{BR}}^{1}=11.2$ and $\alpha_{\mathrm{BR}}=0.3$, for ZMA $\left(E_{\mathrm{g}}=1.1 \mathrm{eV}\right) ; J_{\mathrm{SE}}^{1}=25.3, \alpha_{\mathrm{SE}}=1.64, J_{\mathrm{BR}}^{1}=4.6$, and $\alpha_{\mathrm{BR}}=0$, for CMT $\left(E_{\mathrm{g}} \simeq 0\right) ; J_{\mathrm{SE}}^{1}=4$, and $\alpha_{\mathrm{SE}}=2.58$ (Ref. [4]), $J_{\mathrm{BR}}^{1}=2$, and $\alpha_{\mathrm{BR}}=0.92$, for CMT $\left(E_{\mathrm{g}}=1.6 \mathrm{eV}\right)$.

In conclusion, our results confirm the theoretical prediction that superexchange is the main exchange mechanism at short distances [4], but additionally it may also be stated that the role of the BR-type mechanism of interaction is not negligible and becomes dominant at larger distances.

\section{References}

[1] J.M. Denissen, S. Dakun, K. Kopinga, W.J.M. de Jonge, H. Nishihara, T. Sakakibara, T. Goto, Phys. Rev. B 36, 5316 (1987).

[2] C.J.M. Denissen, H. Nishihara, J.C. van Gool, W.J.M. de Jonge, Phys. Rev. B 33, 7637 (1986).

[3] G.C. de Vries, E. Frikkee, R.B. Helmholdt, K. Kopinga, W.J.M. de Jonge, Physica B 156-157, 321 (1989).

[4] B.E. Larson, K.C. Hass, H. Ehrenreich, Phys. Rev. B 37, 4137 (1988).

[5] D.J.S. Beckett, S.F. Chebab, G. Lamarche, J.C. Woolley, J. Magn. Magn. Mat. 69, 311 (1987).

[6] H. Bednarski, J. Cisowski, to be published.

[7] A. Twardowski, H.J.M. Swagten, W.J.M. de Jonge, Phys. Rev. B 36, 7013 (1987).

[8] W.J.M. de Jonge, J. Magn. Magn. Mat. 100, 322 (1991).

[9] S. Oseroff, P.H. Keesom, in: Diluted Magnetic Semiconductors, in series Semiconducors and Semimetals, Vol. 25, Eds. J.K. Furdyna, J. Kossut, Academic Press, Boston 1988, p. 183. 